\title{
BMJ Open Incidence of chronic wounds in Singapore, a multiethnic Asian country, between 2000 and 2017: a retrospective cohort study using a nationwide claims database
}

\author{
Orlanda Q Goh (D) , ${ }^{1,2,3,4}$ Ganga Ganesan, ${ }^{1}$ Nicholas Graves, ${ }^{5}$ Yi Zhen Ng, ${ }^{6}$ \\ Keith Harding, ${ }^{6}$ Kelvin Bryan Tan ${ }^{1,7}$
}

To cite: Goh 0Q, Ganesan G, Graves N, et al. Incidence of chronic wounds in Singapore, a multiethnic Asian country, between 2000 and 2017: a retrospective cohort study using a nationwide claims database. BMJ Open 2020;10:e039411. doi:10.1136/ bmjopen-2020-039411

- Prepublication history and additional material for this paper are available online. To view these files, please visit the journal online (http://dx.doi org/10.1136/bmjopen-2020039411).

Received 19 April 2020 Revised 07 July 2020 Accepted 06 August 2020

Check for updates

(c) Author(s) (or their employer(s)) 2020. Re-use permitted under CC BY-NC. No commercial re-use. See rights and permissions. Published by BMJ.

For numbered affiliations see end of article.

Correspondence to

Dr Orlanda Q Goh;

orlanda.goh@gmail.com

\section{ABSTRACT}

Objectives Chronic wounds are common, costly and impair quality of life, yet epidemiological data are scarce. We aimed to estimate the incidence trend of a multiethnic Asian population.

Design Retrospective cohort study.

Setting Singapore's nationwide claims database. Participants Singaporeans and permanent residents. Outcomes Patients were identified by International Classification of Disease, Ninth Revision, Australian Modification (ICD-9-AM) and ICD-10-AM codes from al admissions between 2000 and 2017, and categorised according to aetiology: venous, arterial, diabetic and pressure. Comorbidities were extracted from a national database of Charlson Comorbidity Index scores.

Results Between 2000 and 2017, 124023 wound-related claims among 86631 patients were identified. Age-specific rate (ASR) and age-adjusted incidence rates of all wounds increased over 18 years, with greatest increases among those aged $\geq 80$. In 2017 , the median age of patients was 74 (IQR 63-84). Half were male (51\%). 70\% were ethnic Chinese, 15\% Malay and 9\% Indian. In 2017, the crude incidence rate (CIR) was 15 per 100000 persons $(95 \% \mathrm{Cl}$ 14 to 16) for venous wounds, 56 (95\% Cl 53 to 58) for arterial, 168 (95\% Cl 164 to 173) for diabetic and 183 (95\% Cl 179 to 188) for pressure wounds. The CIR of any chronic wound was 296 (95\% Cl 291 to 301). ASRs were greatest in patients aged $\geq 80$ : 92 (95\% Cl 74 to 112) for venous, 478 (95\% Cl 436 to 522) for arterial, 1791 (95\% Cl 1710 to 1876) for diabetic, 3647 (95\% Cl 3530 to 3766) for pressure and 4277 (95\% Cl 4151 to 4407) for any wound. Compared with the Chinese, Indians had thrice the ASRs of venous and arterial wounds and double the ASR of diabetic wounds. Malays had double the ASRs of arterial and diabetic wounds.

Conclusions Chronic wounds are common in the elderly with significant ethnic disparities in this Asian cohort. With the incidence expected to rise with ageing populations, it is crucial to address health disparities and evaluate utilisation and cost to inform clinical practice and health policy.
Strengths and limitations of this study

- Pragmatic and replicable use of a nationwide database to analyse the incidence of wounds in the general population.

- Thorough case identification through including admissions with wounds as a secondary diagnosis.

- Analysis of trends over 18 years.

- Excludes patients who may have used only outpatient services for wounds.

- Subject to systematic errors in estimating incidence based on ICD coding as an upgrade in ICD codes was implemented during the period of observation.

\section{INTRODUCTION}

Chronic wounds are associated with pain, depression, anxiety, embarrassment, functional impairment and reduced quality of life for both patients and caregivers. ${ }^{12}$ They impose extra costs for inpatient and outpatient health services. ${ }^{134}$ However, the magnitude of the problem is poorly understood due to under-reporting and challenges in case identification as they are frequently documented as a comorbid condition. ${ }^{5}$ Chronic wounds are commonly defined as barrier defects that have not healed in 3 months. ${ }^{6}$ The four most common chronic wounds are arterial insufficiency ulcers, venous ulcers, diabetic ulcers and pressure ulcers. ${ }^{7}$ In developed countries, an estimated one to two per cent of the population will experience a chronic wound during their lifetime. ${ }^{8}$ With the absolute incidence of chronic wounds expected to increase in ageing populations with comorbid conditions such as diabetes, this is a silent epidemic that will affect a growing proportion of the global population. ${ }^{5910}$

To reduce the burden of chronic wounds, information on the epidemiology of chronic 
wounds is required to inform decision-making by health service planners. ${ }^{11} 12$ Unfortunately, the reported prevalence of chronic wounds varies widely and ranges from $0.15 \%$ to $1.69 \% .{ }^{13}$ Estimates on the prevalence and incidence of chronic wounds vary by jurisdiction, study designs and methods for reporting prevalence and incidence rates. ${ }^{9} 1415$ Studies also differ on the duration required for chronicity, ranging from 4 weeks to more than 3 months. ${ }^{16}$

The objective of this paper is to estimate the trend in incidence rates of chronic wounds and its four most common categories in the general population in Singapore using a nationwide administrative claims database. This will facilitate better public allocation of healthcare resources in anticipation of an ageing population. To our knowledge, data on the epidemiology of chronic wounds is sparse in Asia. The incidence trends of chronic wounds among multiethnic Singaporeans of Chinese, Malay and Indian descent will be informative for the rest of Asia.

\section{METHODS}

\section{Study population}

We carried out a cohort study of wounds requiring care in acute hospitals among Singaporeans and permanent residents (PRs) between 2000 and 2017. All Singaporeans are insured by a compulsory national health insurance scheme that covers some portion of all inpatient hospital bills. This identification of cases by International Classification of Diseases (ICD) codes in the national claims database allows a population-based estimate of the incidence of chronic wounds. Data on wounds were identified from the Ministry of Health's (MOH) Central Claims Processing System which includes all admissions of Singapore residents to public and private acute care hospitals.

Chronic wounds can be caused by a wide variety of diseases including inflammatory diseases, infection, malignancy and haematological disease. This study focuses on the four major causes of chronic wounds seen in Singapore which are arterial ulcers, venous ulcers, diabetic ulcers and pressure ulcers. Patients with chronic wounds were identified using diagnostic codes from International Classification of Disease, Ninth Revision, Australian Modification (ICD-9-AM) for the period 2000-2011 and from ICD-10-AM for the period 2012-2017.

Patients were considered to have an incident chronic wound-henceforth referred to as 'wounds' - in a year if their admission included diagnosis codes of any of our aetiological subtypes as a primary or secondary diagnosis (details of diagnosis codes in online supplementary table 1):

Venous ulcers: ICD-9-AM: 4540, 4532, 4591; ICD-10-AM: I83.0, I83.2, I87.0.

Arterial insufficiency ulcers: ICD-9-AM: 44023, 44024; ICD-10-AM: I70.23, I70.24.

Diabetic ulcers: ICD-9-AM: 7071, 7078, 7079, 7854 है one of 25070, 25071, 25072 or 25073; ICD-10-AM: E10.73,
E11.73, E13.73, E14.73, E10.52, E11.52, E13.52, E14.52, E09.02, E09.52, E10.69, E11.69, E13.69, E14.69.

Pressure ulcers: ICD-9-AM: 7070; ICD-10-AM: L89.0, L89.1, L89.2, L89.3, L89.4, L89.5, L89.6, L89.7, L89.8, L89.9.

\section{Study variables}

The demographic characteristics of patients were extracted from the MOH Central Claims Processing System. The Charlson Comorbidity Index (CCI) is a summary score of an individual's comorbidities based on the presence of and severity of common chronic diseases. It is commonly used as a prognostic indicator and predicts 10-year survival risk and hospital utilisation in patients with multiple comorbidities. ${ }^{1718}$ The CCI of patients with incident wounds was calculated based on pre-existing comorbidities identified from diagnostic codes obtained from MOH's nationwide administrative databases on inpatient admissions, day surgery and emergency department episodes, public sector primary healthcare clinic visits and subsidised general practitioner clinic visits via the Community Health Assist Scheme. The prevalence of comorbidities among the cohort of patients with incident wounds in 2017 was defined as being diagnosed with any of the comorbidities included in the CCI by the end of 2017.

\section{Statistical analysis}

The demographic and clinical characteristics of patients with different incident wound types in 2017 were compared. Differences in the median age and CCI score between those with and without one of the four specific wound types were tested for statistical significance using the Mann-Whitney U test. Differences in gender, race and prevalence of comorbidities between those with and without a specific wound type were compared using the $\chi^{2}$ test. Differences in demographic or clinical characteristics between individuals with different incident wound types could not be tested for statistical significance as these groups were not mutually exclusive and there were individuals with multiple incident wound types in a year.

The primary outcome was the incidence of any wound, and each of the four aetiological types of wounds, per 100000 persons in the general population. We considered that the different wound types share risk factors and patients may present with coexisting wound types. Hence, patients who presented with more than one wound type in a year were counted as having multiple wounds. To estimate the yearly crude incidence rate (CIR) of chronic wounds in the general population, each admission for a wound type was counted once in a year. Multiple admissions for the same wound type in a patient were counted once in a year only. The denominator for each year was the total mid-year population of Singaporeans and permanent residents obtained from the Department of Statistics of Singapore.

We estimated the CIR and age-adjusted rate (AAR) of all wound types in 2017. The AAR was estimated based 
on the Singapore population in 2000. We also studied the trend of age-specific incidence rates (ASR) of various wound types for the following age groups: <40, 40-49, $50-59,60-69,70-79$ and $\geq 80$. ASR and overall CIR were stratified by the three main ethnic groups in Singapore: Chinese, Malay and Indian. To determine the trend of annual incidence rates during this 18 -year period, CIR and AAR for wounds were estimated. Poisson regression was used to identify the $95 \% \mathrm{CI}$ of all incidence rates.

As there is no consensus on the minimum duration needed to satisfy chronicity and no data on the duration of wounds in administrative databases, neither point nor period prevalence was estimated in our study. All analyses were performed using Stata V.16 (Stata Statistical Software: Release 16. College Station, Texas, USA: StataCorp LLC).

\section{Ethical standards}

As this study was conducted in accordance with the national guidelines for the use of administrative databases and personal data protection using anonymised data from the Singapore MOH's administrative databases, the approval of an ethical committee was not required for this study.

\section{Patient and public involvement}

As this was a retrospective analysis of an anonymised national claims database, patients and the public were not involved in the design or conduct of this study. A summary of the results will be shared with the public by the $\mathrm{MOH}$.

\section{RESULTS}

\section{Patient characteristics}

The average population size of Singaporeans and PRs during this 18-year period was 3645514. Between 2000 and 2017, 124023 wound-related admissions of 86631 patients were identified. There were 11733 patients admitted with a wound-related diagnosis in 2017. The median age of patients in 2017 was 74 (IQR 63-84). Approximately half of the patients were male $(51 \%)$ and the majority were Chinese (70\%). The median CCI score was 6 (IQR 3-9). The prevalence of conditions associated with end-organ damage, such as congestive heart failure, chronic obstructive pulmonary disease, acute myocardial infarction, stroke, dementia and moderate to severe kidney disease ranged from $20 \%$ to $47 \%$ in this population (table 1).

\section{Incidence trends}

There was an increase in the yearly CIRs of venous, arterial, diabetic, pressure, multiple and any wounds in the general population during this 18-year duration. This trend was also seen in the respective AAR adjusted to the population structure in 2000 (figure 1A,B and table 2).

There was a sharp increase in the AAR of any wound in 2012 and 2013, which was contributed mainly by the sharp increases in AAR of pressure and diabetic wounds in the same years. Nevertheless, the graph shows an increasing secular trend of AAR from 2012 to 2017.

The incidence rates of venous, arterial, diabetic and pressure wounds increased exponentially with age. This increase is highest among those aged 80 and older. This is shown in the graphs trending the ASR of wounds in years 2000, 2005, 2010, 2015 and 2017 (figure 2A-E).

\section{Incidence rates in 2017}

In 2017, the CIR of chronic wounds per 100000 persons in the general population was 15 (95\% CI 14 to 16) for venous wounds, 56 (95\% CI 53 to 58) for arterial wounds, 168 (95\% CI 164 to 173) for diabetic wounds and 183 (95\% CI 179 to 188) for pressure wounds. Further, 114 per 100000 persons (95\% CI 110 to 117) in the general population presented with more than one wound type or had multiple wounds. In total, 296 per 100000 persons (95\% CI 291 to 301) in the general population, and 792 (95\% CI 777 to 807) per 100000 persons aged 50 and older in the general population had any chronic wound (table 3).

Diabetic and pressure wounds were the most common wounds in each age group. All four wound types had an increasing incidence with age. Among those aged 80 and older in the general population, the ASR was 92 per 100000 persons (95\% CI 74 to 113) for venous wounds, 478 (95\% CI 436 to 522) for arterial wounds, 1791 (95\% CI 1710 to 1876) for diabetic wounds, 3647 (95\% CI 3530 to 3766) for pressure wounds and 4277 (95\% CI 4151 to $4407)$ for any wound. In the highest age group, patients aged 80 and older, the ASR of arterial and diabetic wounds were approximately twofold higher than that of those aged 70-79. The age group-specific incidence rate of pressure wounds among those aged 80 and older was more than fourfold higher compared with those aged 70-79 (table 3, figure 3).

Significant differences in incidence rates were also observed among the major ethnic groups. Among those aged 50 and older in the general population, the ASR was 38 per 100000 persons (95\% CI 35 to 42 ) for venous wounds, 150 per 100000 persons (95\% CI 144 to 156) for arterial wounds, 446 per 100000 persons (95\% CI 435 to 457) for diabetic wounds and 505 per 100000 persons (95\% CI 493 to 517) for pressure wounds. Compared with the Chinese majority, Indians had threefold higher incidence rates of venous and arterial wounds and a twofold higher incidence rate of diabetic wounds. Compared with the Chinese majority, Malays had twofold higher incidence rates of arterial and diabetic wounds. These differences are seen among those aged 50 and older, and also in the general population (table 3 ). These relative differences in incidence rates of wounds among the three ethnic groups were consistent over the 18-year period. Malays and Indians had higher incidences of venous, arterial and diabetic wounds, whereas the Chinese had the highest incidence of pressure wounds (see online supplementary figure 1A-D). 
Table 1 Characteristics of patients who had an incident wound in 2017

\begin{tabular}{|c|c|c|c|c|c|}
\hline & All & Venous & Arterial & Diabetic & Pressure \\
\hline No. of individuals* & 11733 & 598 & 2207 & 6679 & 7267 \\
\hline Age, median (IQR)† & $74(63-84)$ & $66(57-76)$ & $68(60-78)$ & $70(60-81)$ & $80(70-87)$ \\
\hline \multicolumn{6}{|l|}{ Race† } \\
\hline Chinese, n (\%) & 8255 (70.4) & $354(59.2)$ & $1295(58.7)$ & $4129(61.8)$ & $5800(79.8)$ \\
\hline Indian, n (\%) & $1113(9.5)$ & $112(18.7)$ & $332(15.0)$ & $854(12.8)$ & $360(5.0)$ \\
\hline \multicolumn{6}{|l|}{ Comorbidities } \\
\hline DM, n (\%)† & 7766 (66.2) & $261(43.7)$ & $1293(87.1)$ & $6500(97.3)$ & $3886(53.5)$ \\
\hline PVD, n (\%)† & $3579(32.6)$ & $175(38.5)$ & $2153(99.7)$ & $2921(44.9)$ & 1029 (15.2) \\
\hline CTD, n (\%)† & $315(2.9)$ & $35(7.7)$ & $63(2.9)^{\#}$ & $133(2.1)$ & $206(3.0)^{\#}$ \\
\hline COPD, n (\%)† & $2143(19.5)$ & $110(24.2)$ & $387(17.9)$ & $1121(17.3)$ & 1389 (20.5) \\
\hline Stroke, n (\%)† & 4325 (39.3) & $103(22.6)$ & $752(34.8)$ & $2410(37.1)$ & $3220(47.5)$ \\
\hline Dementia, n (\%)† & 2627 (23.9) & $33(7.3)$ & $220(10.2)$ & 1149 (17.7) & $2380(35.1)$ \\
\hline MS liver cirrhosis, $\mathrm{n}(\%) \ddagger$ & $578(4.9)$ & $33(5.5)^{\#}$ & $87(3.9)$ & $333(5.0)^{\#}$ & $412(5.7)$ \\
\hline MS CKD, n (\%)† & $5145(46.8)$ & $135(29.7)$ & $1378(63.8)$ & 3661 (56.3) & $2963(43.7)$ \\
\hline Metastatic cancer, n (\%)† & 976 (8.9) & $16(3.5)$ & $73(3.4)$ & 406 (6.3) & $834(12.3)$ \\
\hline $\mathrm{CCl}$ score, median (IQR)† & $6(3-9)$ & $3(1-6)$ & 7 (5-9) & 7 (5-9) & $6(3-9)$ \\
\hline
\end{tabular}

${ }^{*}$ Non-additive across this row as one patient may have multiple wound diagnoses. Statistical significance of differences in median age and $\mathrm{CCl}$ score was tested using the Mann-Whitney $U$ test. Statistical significance of differences in percentages was tested using the $\chi^{2}$ test. $\dagger \mathrm{p}<0.01$ when compared with those without the specified wound, except for those marked with \#. $\ddagger \mathrm{p}<0.05$ when compared with those without the specified wound, except for those marked with \#. $\mathrm{AMI}$, acute myocardial infarction; $\mathrm{CCl}$, Charlson Comorbidity Index; CHF, congestive heart failure; CKD, chronic kidney disease; COPD, chronic obstructive pulmonary disease; CTD, connective tissue disease; CVA, cerebrovascular disease; DM, diabetes mellitus; MS, moderate to severe; PVD, peripheral vascular disease.

\section{DISCUSSION}

In this comprehensive population-based study of nationwide claims for admissions to acute hospitals among all Singaporeans and PRs, we estimated the incidence of wounds in the general population over 18 years and showed an increasing trend of venous, arterial, diabetic and pressure wounds. In 2017, the incidence of any wound was 300 per 100000 persons in the general population and 800 per 100000 among those aged 50 and older. To our knowledge, this is the first report of incidence rates in the general population in Asia. Outside of Asia, Heyer and colleagues have reported national incidence rates based on a German administrative database. ${ }^{9}$ Other reports on the incidence of wounds have been limited to selected populations such as those with diabetes or institutionalised patients. Specific epidemiological data on the individual wounds types are frequently inadequate and studies that have reported on the incidence of chronic wounds are seldom comparable due to the heterogeneity of the study design and methodology. ${ }^{1315} 1619$

The incidence has consistently increased with age, with the sharpest rise observed in the oldest age group of those aged 80 and above. The global burden of disease is set to rise with ageing populations as better management of infectious disease, cardiovascular disease and cancer prolong survival and increase the prevalence of the frail elderly. Interactions between the increasing burden of comorbid diseases such as metabolic syndrome, vascular risk factors and end-organ damage, and frailty in the elderly increase the incidence of wounds. ${ }^{9}$ Furthermore, age is also a predictor of poor wound healing. ${ }^{10}$ The trends reported in this study lend more evidence to the prediction that the burden of chronic wounds in ageing populations is a 'snowballing threat' to public health and the economy. ${ }^{5}$

This highlights the global need to estimate local incidence rates of this neglected epidemic. Although not immediately fatal, wounds result in significant morbidity through pain, impaired self-image and restricted function especially when located at the lower limbs. ${ }^{12}$ Wounds tend to be recurrent and result in high resource use in a complex care setting, requiring medications, revascularisation procedures, prolonged antibiotic therapy, specialised dressing and nursing care, readmissions, extended length of stay and frequent outpatient visits. In addition, there is direct and indirect loss of productivity through 


\section{A}

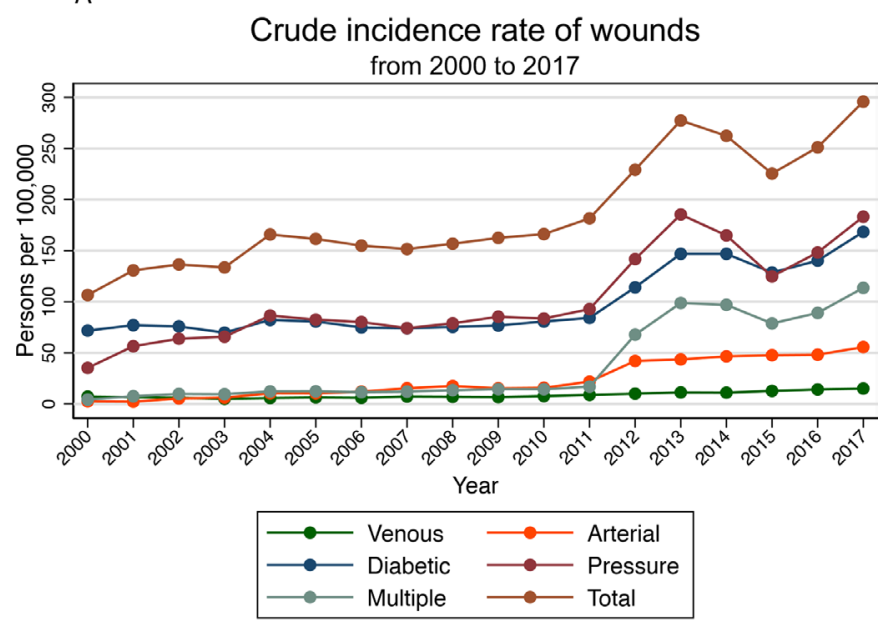

B

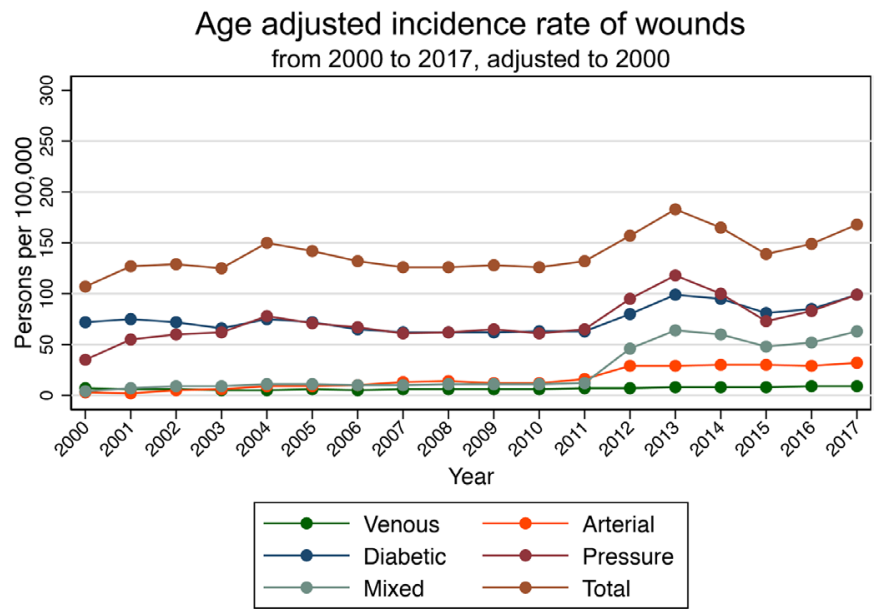

Figure 1 Trends of incidence rates from 2000 to 2017. (A) The trend of CIR of wounds from 2000 to 2017. (B) The trend of AAR of wounds from 2000 to 2017 . The incidence rates of specific wound types do not add up to the total incidence rates as one patient may have multiple wound diagnoses in the same year.

loss of function and significant caregiver burden. ${ }^{20}$ The management of wounds is thus a complex multidisciplinary care challenge. In order to begin defining the magnitude of this problem, health systems will benefit from monitoring years lived with disability rates of wounds alongside other common conditions studied in the Global Burden of Disease Study. ${ }^{21}$

This has implications for healthcare systems. In highresource healthcare systems, this is an opportunity to lower the incidence rates of wounds by improving health financing and delivery of integrated care for better prevention and management of risk factors that include well-reported diseases like diabetes and hypertension, as well as the less reported syndrome of frailty among the geriatric population or the chronically sick. Low-resource countries may benefit from early recognition of this problem and investing early in a healthcare system that is poised to mitigate the morbidity and costs associated with the wounds epidemic.
Understanding ethnic disparities is crucial to identifying targeted measures to reduce chronic wounds. We reported that venous, arterial and diabetic wounds are more common among the Malays and Indians compared with the Chinese majority. This may partly be due to inherent genetic differences in risk profile. The prevalence of chronic venous disease, a precursor of venous wounds, manifested by spider veins, varicose veins, trophic changes, oedema and a history of venous thrombosis, has been shown to be influenced by ethnicity. ${ }^{22}$ At the same time, our results are consistent with the higher incidence of lower extremity amputations, a late complication of arterial and diabetic wounds, among Malays and Indians in Singapore. ${ }^{23}$ More research is needed to determine the extent to which disparities are mediated by differences in the control of known risk factors, socioeconomic status and access to health services, so as to inform interventions that will aggressively mitigate these factors.

The CIR of wounds of 300 per 100000 persons in the general population in 2017 is similar to the rate of 280 previously reported in Germany in 2012 by Heyer and colleagues. ${ }^{9}$ Our estimated CIR of venous wounds of 15 per 100000 persons is close to the range of 20-350 per 100000 previously reported in an international study in 1999. ${ }^{24}$ However, it is one-quarter of that estimated by Heyer and colleagues in their more recent study. This discrepancy could be due to inherent ethnic differences in the prevalence of risk factors for venous wounds, which has been shown to be more common among nonHispanic whites compared with Asians. ${ }^{22}$

The arterial wound rate of 60 per 100000 persons and diabetic wound rate of 170 per 100000 persons are twice that reported in the German setting, which is the only other study that has reported these rates in the general population. ${ }^{15}$ Arterial and diabetic wounds share a common pathophysiological process and are considered late-stage manifestations of disease. In our population, $87 \%$ of those with an arterial wound have comorbid diabetes. The higher rates of arterial and diabetic wounds in our population are consistent with existing evidence of higher diabetes prevalence in Singapore and poorer outcomes of diabetic patients in Singapore compared with Organisation for Economic Co-operation and Development (OECD) nations. In 2017, the age-sex standardised rate of lower extremity amputations among diabetics in Singapore, a late complication of arterial and diabetic wounds, exceeded all OECD countries, with the exception of Israel and Mexico. ${ }^{25}$ In response to the alarmingly poor outcomes of our patients with diabetes, the Singaporean government has declared a 'war on diabetes' and initiated a multi-ministerial task force to improve the quality of care of diabetes through campaigning to encourage better compliance to lifestyle modifications, enhancing the skills of healthcare providers, empowering the patient community and increasing health financing of care for diabetes. ${ }^{26}$

The pressure wound incidence rate of 180 per 100000 persons is also twice that reported in the German setting. 

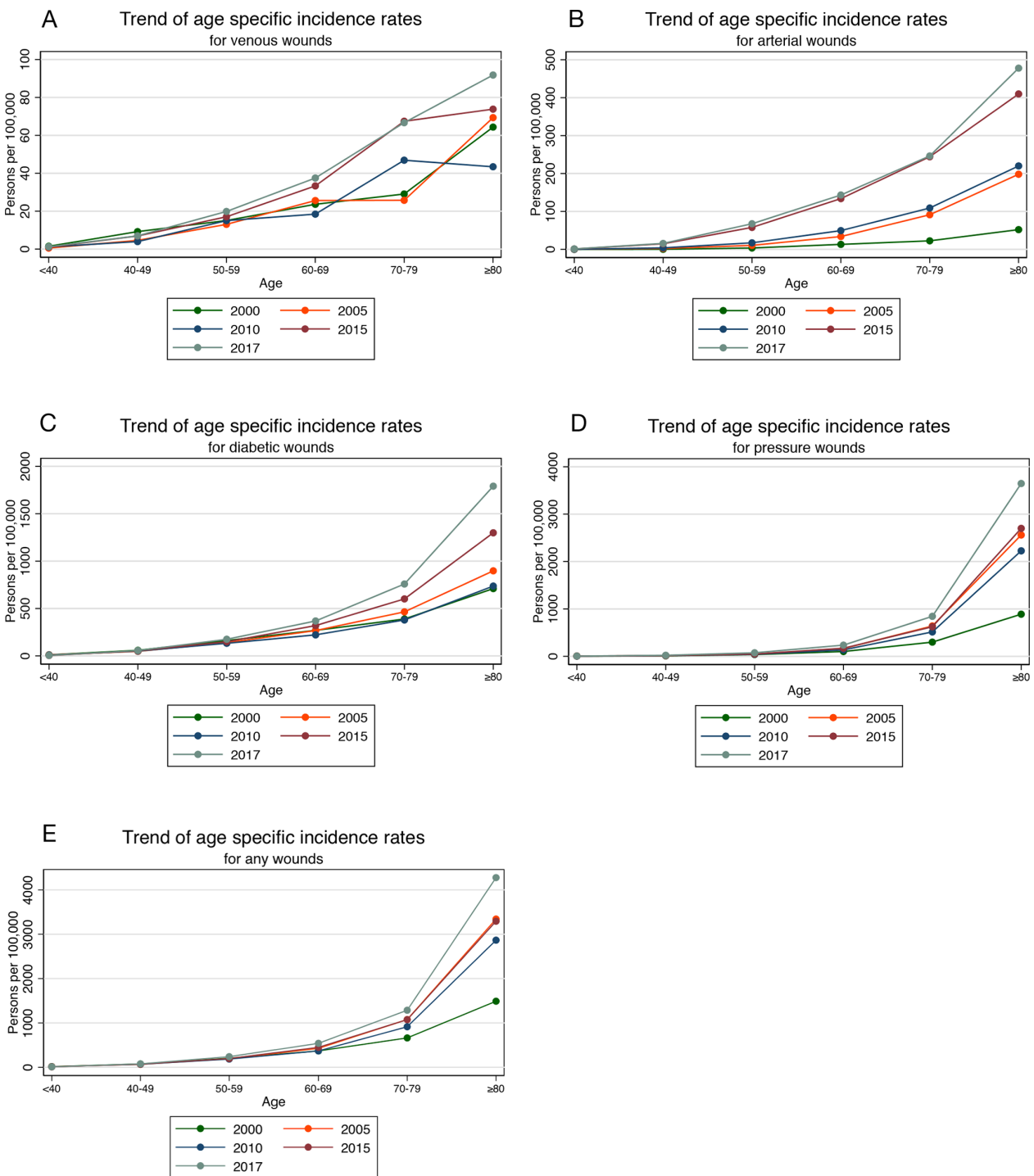

Figure 2 Trends of ASR of wound types from 2000 to 2017. (A) The increases in ASR of venous wounds from 2000 to 2017. (B-E) Similar trends for arterial, diabetic, and pressure wounds and total incidence, respectively.

Again, there were also no other studies in the general population with which we could make a direct comparison. One-year incidence rates reported among patients in cohort studies across various hospital settings ranged from $11 \%$ to $18 \% .{ }^{27}{ }^{28}$ Since the incidence of pressure wounds is higher among elderly, functionally dependent, intensive care unit and institutionalised patients, more information is needed on the risk profile of the population to explain this difference. ${ }^{15}$

The strengths of this study include the use of the nationwide claims database to access all inpatient utilisation episodes and the long duration of observation, allowing us to reliably make a population estimate and trend the incidence across 18 years. By including secondary diagnoses of wounds, we accounted for wounds which are frequently under-reported as they tend to be regarded as comorbid condition by clinicians. Although there is a limit to the generalisability of incidence rates to other populations due to differences in the age structure, ethnicity, disease epidemiology and healthcare infrastructure, the trend of increasing rates can be generalised to other countries with an ageing population. The method of identifying cases through inpatient utilisation is reliable and useful for future research. It is a pragmatic method that can be easily replicated in other countries for comparison, especially in low-resource settings where setting up a registry is not feasible. Inpatient utilisation is the most significant contributor to direct healthcare costs. These population-based estimates may be used to build on recently published estimates on the incidence and costs of wounds in the second largest healthcare institution in Singapore, to derive the economic burden of wounds to the population. ${ }^{29}$ This will provide valuable information to inform future cost-effectiveness evaluations of interventions to reduce the frequency, duration and recurrence of wounds for decision-making and priority setting. ${ }^{30}$

Our study has important limitations. The complete reliance on ICD codes to identify cases subjected the data to fluctuations related to a change from the use of ICD-9-AM to ICD-10-AM in 2012. There were sharp increases in the 


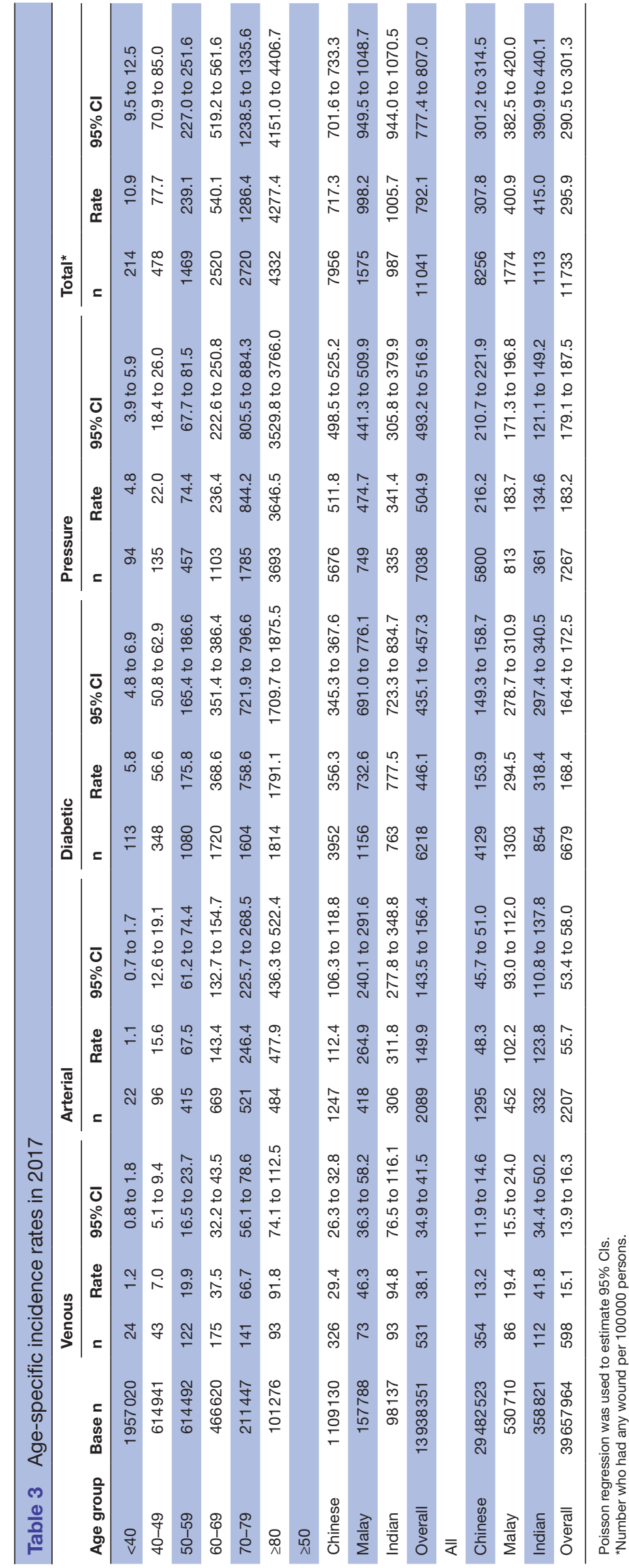


Incidence of wounds by type and age groups in 2017

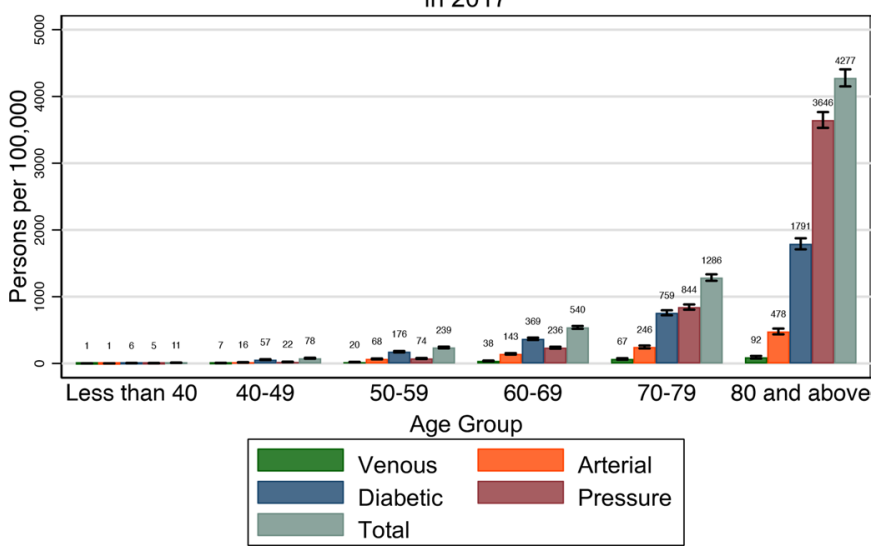

Figure 3 Incidence of different wounds by age group in 2017. The incidence rates of specific wound types do not add up to the total incidence rates as one patient may have multiple wound diagnoses in the same year.

trend of pressure and diabetic wounds seen in 2012. This could have been related to the use of more specified codes for diabetic and pressure wounds in ICD-10-AM. Since codes were not more inclusive but more detailed and specific, this may have influenced hospital coders in the medical records office to pay more attention to these diagnoses when transiting from ICD-9-AM to ICD-10-AM (see online supplementary table 1). Regardless of the spike in rates seen in 2012 and 2013, our figures show an increasing trend in age-adjusted rates for all wound types.

Dependence on inpatient utilisation as a surrogate for wound incidence might overestimate incidence rates in recent years. The increase in rates in most recent years could be contributed by an increase in patient awareness and utilisation of inpatient health services for therapeutic management such as revascularisation procedures and advanced wound nursing care options rather than a true increase in incidence. Furthermore, case identification through diagnosis coding without supplementation with other criteria may also result in an overestimation. ${ }^{9}$ Overall, these effects may be minimal as there could be a concurrent underestimation of incidence through the exclusion of patients with wounds who only used outpatient services. Nonetheless, we expect that in spite of these uncertainties, there is a true increase in incidence due to the effects of an ageing population in a high-resource healthcare setting for reasons mentioned earlier.

We were unable to determine the duration of wounds to quantify chronicity. This is not possible based on administrative data and makes the case for the establishment of a wounds registry to more accurately estimate the incidence and prevalence of wounds in the general population. However, the high administrative burden of establishing a registry may not be feasible in low-resource healthcare systems.

Our study estimated the incidence rate of wounds and four aetiological types in the general population based on a national administrative database over an 18-year period.
We found an increasing trend across all wound types, with the highest increase among the oldest age groups. The burden of wounds is expected to increase as the population ages. Our findings suggest that countries should actively monitor the epidemiology of wounds to better quantify the burden of disease so that the data can be used for priority setting for health services delivery and for evaluating the cost-effectiveness of targeted interventions. Future analyses might build on this work to estimate the economic burden of chronic wounds.

\section{Author affiliations}

${ }^{1}$ Policy and Research Division, Ministry of Health, Singapore

${ }^{2}$ Department of Internal Medicine, Singapore General Hospital, Singapore ${ }^{3}$ SingHealth Duke-NUS Medicine Academic Clinical Programme, SingHealth DukeNUS Academic Medical Centre, Singapore

${ }^{4}$ Johns Hopkins Bloomberg School of Public Health, Baltimore, United States ${ }^{5}$ Health Services \& Systems Research, Duke-NUS Medical School, Singapore ${ }^{6}$ Wound Care Innovation for the Tropics Programme, Skin Research Institute of Singapore, $A^{*}$ STAR, Singapore

${ }^{7}$ Saw Swee Hock School of Public Health, National University of Singapore, Singapore

Contributors $\mathrm{OG}, \mathrm{GG}$ and $\mathrm{KT}$ conceived and designed the study. $\mathrm{OG}$ did the statistical analysis and drafted the report. GG and KT contributed to the statistical analysis. OG, GG, NG, YZN, KH and KT contributed to data interpretation, editing of the report and approved the final version before submission.

Funding The authors have not declared a specific grant for this research from any funding agency in the public, commercial or not-for-profit sectors.

\section{Competing interests None declared.}

Patient and public involvement Patients and/or the public were not involved in the design, or conduct, or reporting or dissemination plans of this research.

\section{Patient consent for publication Not required}

Provenance and peer review Not commissioned; externally peer reviewed.

Data availability statement № data are available.

Open access This is an open access article distributed in accordance with the Creative Commons Attribution Non Commercial (CC BY-NC 4.0) license, which permits others to distribute, remix, adapt, build upon this work non-commercially, and license their derivative works on different terms, provided the original work is properly cited, appropriate credit is given, any changes made indicated, and the use is non-commercial. See: http://creativecommons.org/licenses/by-nc/4.0/.

\section{ORCID iD}

Orlanda Q Goh http://orcid.org/0000-0001-8423-2703

\section{REFERENCES}

1 Augustin M, Brocatti LK, Rustenbach SJ, et al. Cost-of-illness of leg ulcers in the community. Int Wound J 2014;11:283-92.

2 Herberger K, Rustenbach SJ, Grams L, et al. Quality-of-care for leg ulcers in the metropolitan area of Hamburg-a community-based study. J Eur Acad Dermatol Venereol 2012;26:495-502.

3 Margolis DJ, Bilker W, Santanna J, et al. Venous leg ulcer: incidence and prevalence in the elderly. J Am Acad Dermatol 2002;46:381-6.

4 Purwins S, Herberger K, Debus ES, et al. Cost-of-illness of chronic leg ulcers in Germany. Int Wound J 2010;7:97-102.

5 Sen CK, Gordillo GM, Roy S, et al. Human skin wounds: a major and snowballing threat to public health and the economy. Wound Repair Regen 2009;17:763-71.

6 Kyaw BM, Järbrink K, Martinengo L, et al. Need for improved definition of "chronic wounds" in clinical studies. Acta Derm Venereol 2018;98:157-8

7 The Wound Healing Society. Chronic wound care guidelines [online]. Available: https://woundheal.org/files/2017/final_pocket_guide_ treatment.pdf [Accessed 9 Dec 2019].

8 Gottrup F. A specialized wound-healing center concept: importance of a multidisciplinary department structure and surgical 
treatment facilities in the treatment of chronic wounds. Am J Surg 2004;187:S38-43.

9 Heyer K, Herberger K, Protz K, et al. Epidemiology of chronic wounds in Germany: analysis of statutory health insurance data. Wound Repair Regen 2016;24:434-42.

10 Wicke C, Bachinger A, Coerper S, et al. Aging influences wound healing in patients with chronic lower extremity wounds treated in a specialized wound care center. Wound Repair Regen 2009;17:25-33.

11 Ward MM. Estimating disease prevalence and incidence using administrative data: some assembly required. J Rheumatol 2013;40:1241-3.

12 Divajeva D, Marsh T, Logstrup S, et al. Economics of chronic diseases protocol: cost-effectiveness modelling and the future burden of non-communicable disease in Europe. BMC Public Health 2014;14:456.

13 Martinengo L, Olsson M, Bajpai R, et al. Prevalence of chronic wounds in the general population: systematic review and metaanalysis of observational studies. Ann Epidemiol 2019;29:8-15.

14 Barendregt JJ, Doi SA, Lee YY, et al. Meta-analysis of prevalence. J Epidemiol Community Health 2013;67:974-8.

15 Graves N, Zheng $\mathrm{H}$. The prevalence and incidence of chronic wounds: a literature review. Wound Pract Res J Aust Wound Manag Assoc 2014;22:4

16 Järbrink K, Ni G, Sönnergren H, et al. Prevalence and incidence of chronic wounds and related complications: a protocol for a systematic review. Syst Rev 2016:5:152.

17 Charlson ME, Pompei P, Ales KL, et al. A new method of classifying prognostic comorbidity in longitudinal studies: development and validation. J Chronic Dis 1987;40:373-83.

18 Low LL, Tay WY, Ng MJM, et al. Frequent hospital admissions in Singapore: clinical risk factors and impact of socioeconomic status. Singapore Med J 2018;59:39-43.

19 Danwang C, Tochie JN, Mazou TN, et al. Contemporary occurrence and aetiology of chronic leg ulcers in Africa: a systematic review and meta-analysis protocol. BMJ Open 2019;9:e026868.

20 Tan B, Tan E, Chong S-J, et al. An economic evaluation of chronic wound management in a tertiary hospital. Wound Pract Res J Aust Wound Manag Assoc 2016;24:130-6.
21 James SL, Abate D, Abate $\mathrm{KH}$, et al. Global, regional, and national incidence, prevalence, and years lived with disability for 354 diseases and injuries for 195 countries and territories, 1990-2017: a systematic analysis for the global burden of disease study 2017. Lancet 2018;392:1789-858.

22 Criqui $\mathrm{MH}$, Jamosmos $\mathrm{M}$, Fronek $\mathrm{A}$, et al. Chronic venous disease in an ethnically diverse population: the San Diego population study. Am $J$ Epidemiol 2003;158:448-56.

23 Ang Y, Chun WY, Saxena N, et al. Diabetes-related lower extremity amputations in Singapore - Yee Ang, Chun Wei Yap, Nakul Saxena, Lee-Kai Lin, Bee Hoon Heng, 2017. Proc Singap Healthc 2017;26:76-80.

24 Clement DL. Venous ulcer reappraisal: insights from an international task force. J Vasc Res 1999;36:42-7.

25 OECD. Organisation for economic co-operation and development major lower extremity amputation in adults with diabetes, 2012 and 2017 (or nearest year) [online], 2017. Available: https://read.oecdilibrary.org/social-issues-migration-health/major-lower-extremityamputation-in-adults-with-diabetes-2012-and-2017-or-nearest-year_ 77b55d01-en [Accessed 12 Dec 2019].

$26 \mathrm{MOH}$. War on diabetes [online]. Available: https://www.moh.gov.sg/ wodcj [Accessed 1 Feb 2020]

27 VanDenKerkhof EG, Friedberg E, Harrison MB. Prevalence and risk of pressure ulcers in acute care following implementation of practice guidelines: annual pressure ulcer prevalence census 1994-2008. J Healthc Qual 2011;33:58-67.

28 Fife C, Otto G, Capsuto EG, et al. Incidence of pressure ulcers in a neurologic intensive care unit. Crit Care Med 2001;29:283-90.

29 Lo ZJ, Lim X, Eng D, et al. Clinical and economic burden of wound care in the tropics: a 5-year institutional population health review. Int Wound J 2020;17:790-803.

30 International guidelines. Pressure ulcer prevention prevalence and incidence in context A consensus document - Wounds International [online], 2009. Available: https://www.woundsinternational.com/ resources/details/international-guidelines-pressure-ulcer-preventionprevalence-and-incidence-in-context-a-consensus-document [Accessed 12 Dec 2019]. 